Supporting Information

\title{
Effect of Morphology Evolution on the Anti-Corrosion Performance of Super-Hydrophobic Surfaces and Lubricant-Infused Surfaces
}

Meiling Zhang ${ }^{a}$, Gaohui Sun ${ }^{a^{*}}$, Hui Guo ${ }^{b}$, Qi Liu ${ }^{a, c^{*}}$, Jingyuan Liu ${ }^{a}$, Jing Yu ${ }^{a}$, Rongrong Chen ${ }^{a, c}$, Liangtian Gao $^{c}$, Jun Wang $^{a}$

a. Key Laboratory of Superlight Materials and Surface Technology, Ministry of Education, College of Materials Science and Chemical Engineering, Harbin Engineering University, 145 Nantong Street, Harbin 150001, China.

b. SD Steel Rizhao Co. Ltd, 1 Lingang Road, Rizhao 276800, China.

c. HIT (Hainan) Military-Civilian Integration Innovation Research Institute Co. Ltd, 1 lehuo Avenue, Yingzhou Town, Lingshui County, Hainan 572400, China

d. College of Shipbuilding Engineering, Harbin Engineering University, 145 Nantong Street, Harbin 150001, China.

Corresponding Author:

Gaohui Sun, E-mail:sungaohui1987@163.com; Qi Liu, E-mail:

qiliu@hrbeu.edu.cn

Number of Pages: 13

Number of Figures: 11 
Number of Tables: 4

Characterization: The surface morphologies and structure were characterized by scanning electron microscopy (SEM) using a JEOL JSM-6480A microscope and transmission electron microscopy (TEM, JEOL JEM-2100). X-ray photoelectron spectroscopy was performed using an X-ray photoelectron spectrometer (XPS; ESCALAB250Xi, USA). The corrosion resistance performance of the samples was analyzed by an IM6, German, Zahner electrochemical workstation. Electrochemical impedance spectroscopy (EIS) was performed using a three-electrode system (the samples served as the working electrode, $\mathrm{Ag} / \mathrm{AgCl}$ served as the reference electrode and a platinum sheet served as the counter electrode).

\section{Contact angle and hysteresis measurements:}

In-air contact angles and hysteresis were measured by a Data Physics OCA20 analysis system. In-air contact angles were recorded 10 s after dropping $5 \mu \mathrm{L}$ of testing liquids on the surface. Water sliding angles were measured by dispensing a $10 \mu \mathrm{L}$ water droplet on the surface and tilting the membrane until the droplet rolled off the surface. The contact angles and sliding angles were the averaged values of 5 measurements of 5 different samples for each sample. 

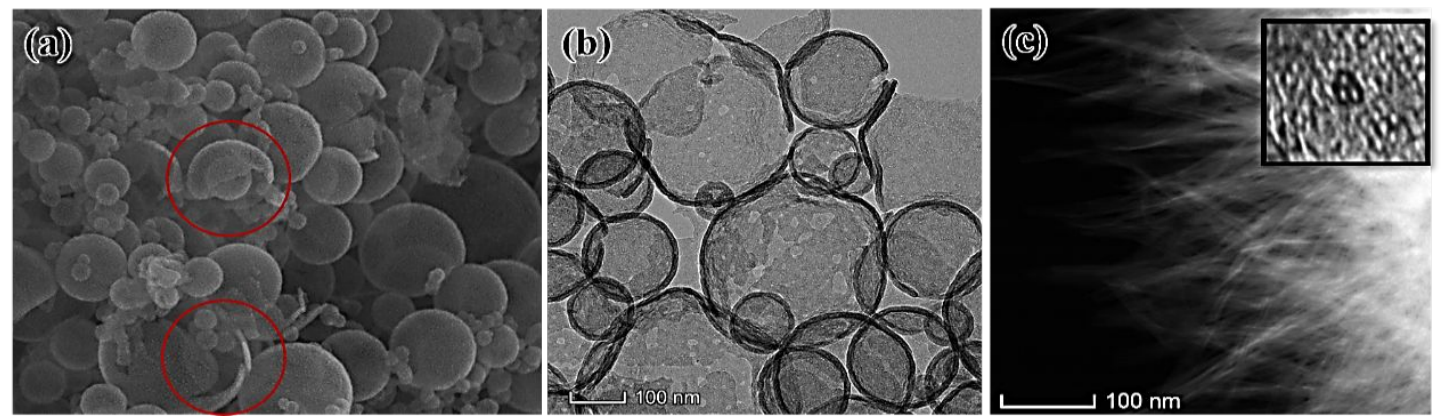

Figure S1 (a) the hollow interior of bayberry-like hollow silica capsules is clearly revealed with some broken sphere. (b) TEM of bayberry-like hollow silica capsules, the shells thickness is about $10 \mathrm{~nm}$. (c) The TEM of urchin-like microspheres, constructed from numerous nanotubes which exhibit uniform nanoscale morphology of crossed tube of 70-90 nm in length. (Inset: the open ends of the nanotubes). Several holes can be observed because of the open ends of the nanotubes. The outer and inner diameters of these holes are estimated to be approximately 7.4 and $3.2 \mathrm{~nm}$, respectively. 


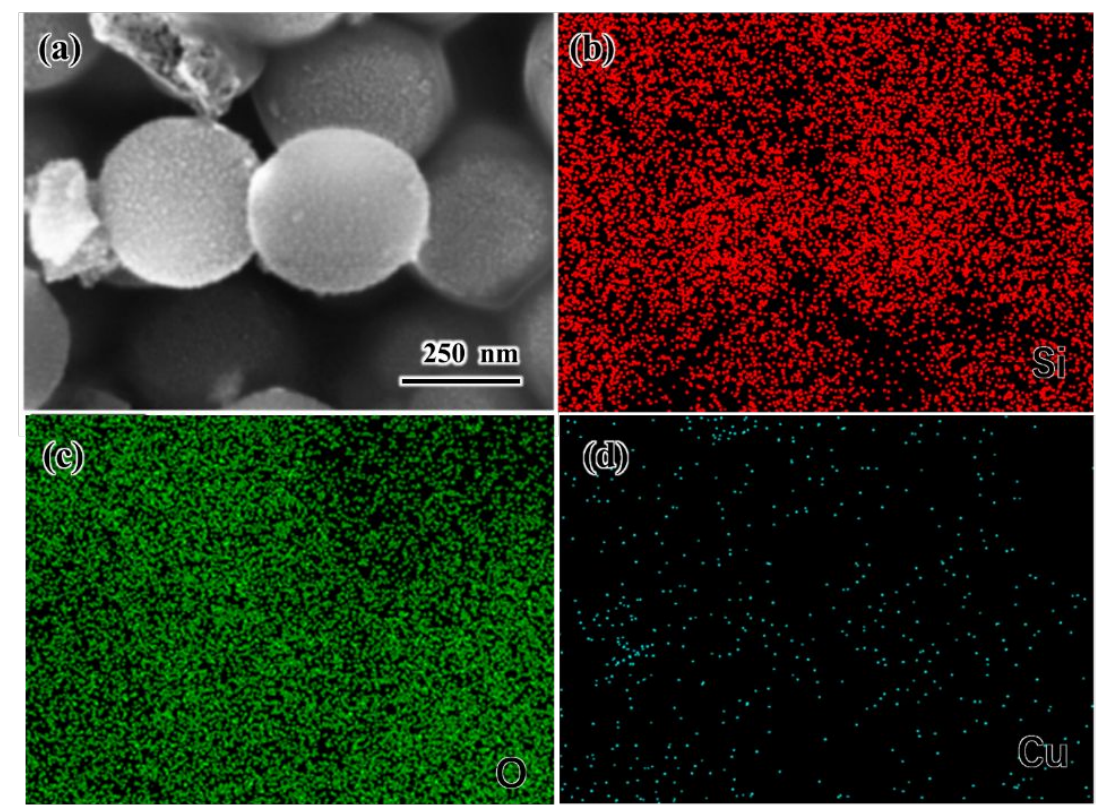

Figure S2 (a) SEM images and (b-d) EDS mappings of the urchin-like microspheres. The energy-dispersive X-ray spectroscopy mapping illustrates the homogeneous distribution of $\mathrm{Si}$ and $\mathrm{O}$ in the bayberry-like hollow silica microspheres. 


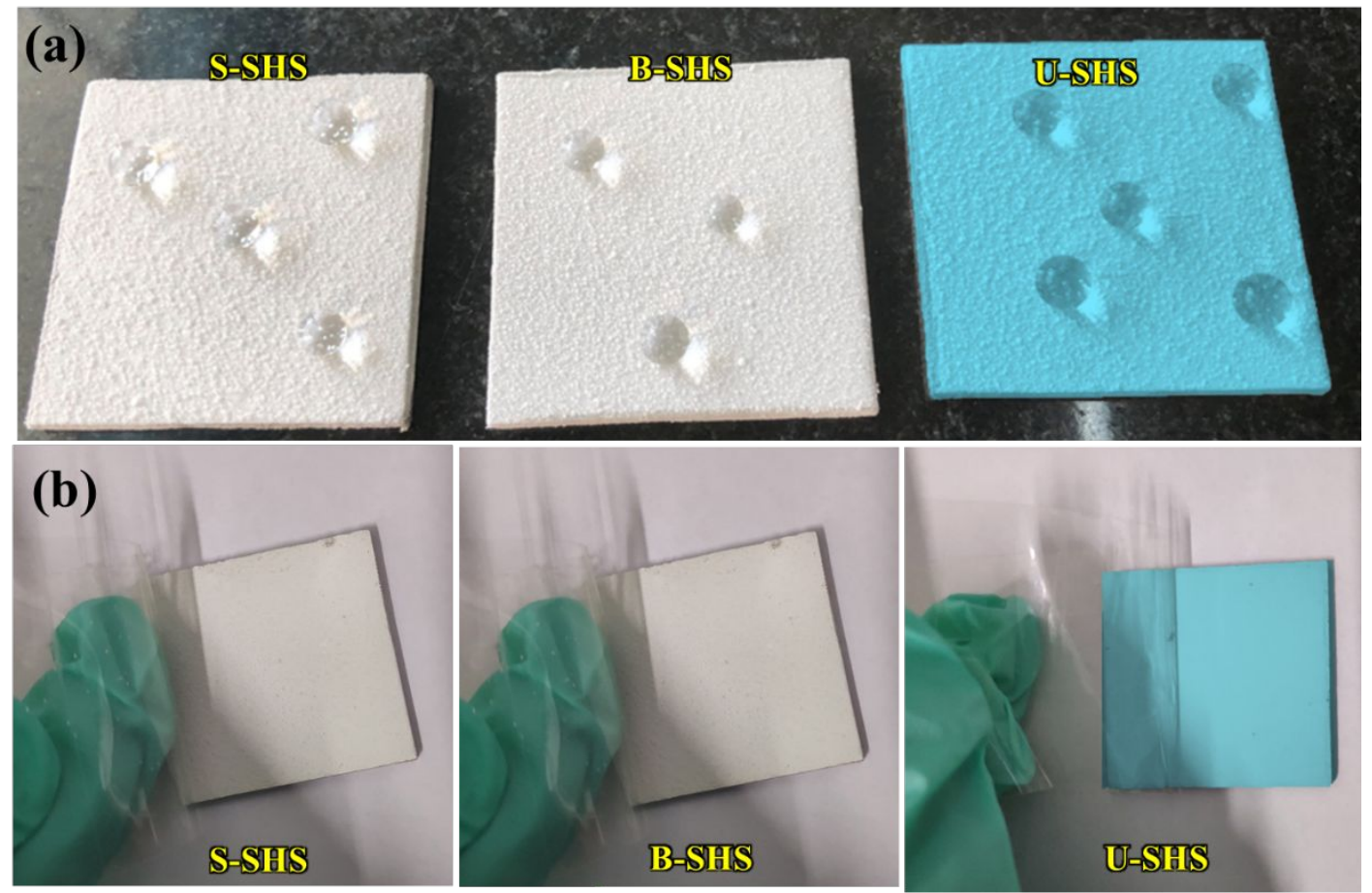

Figure S3 Digital photographs of (a) droplets on three kinds of superhydrophobic surfaces and (b) their peeling experiments. The experimental results show that the samples after spraying have good adhesion 


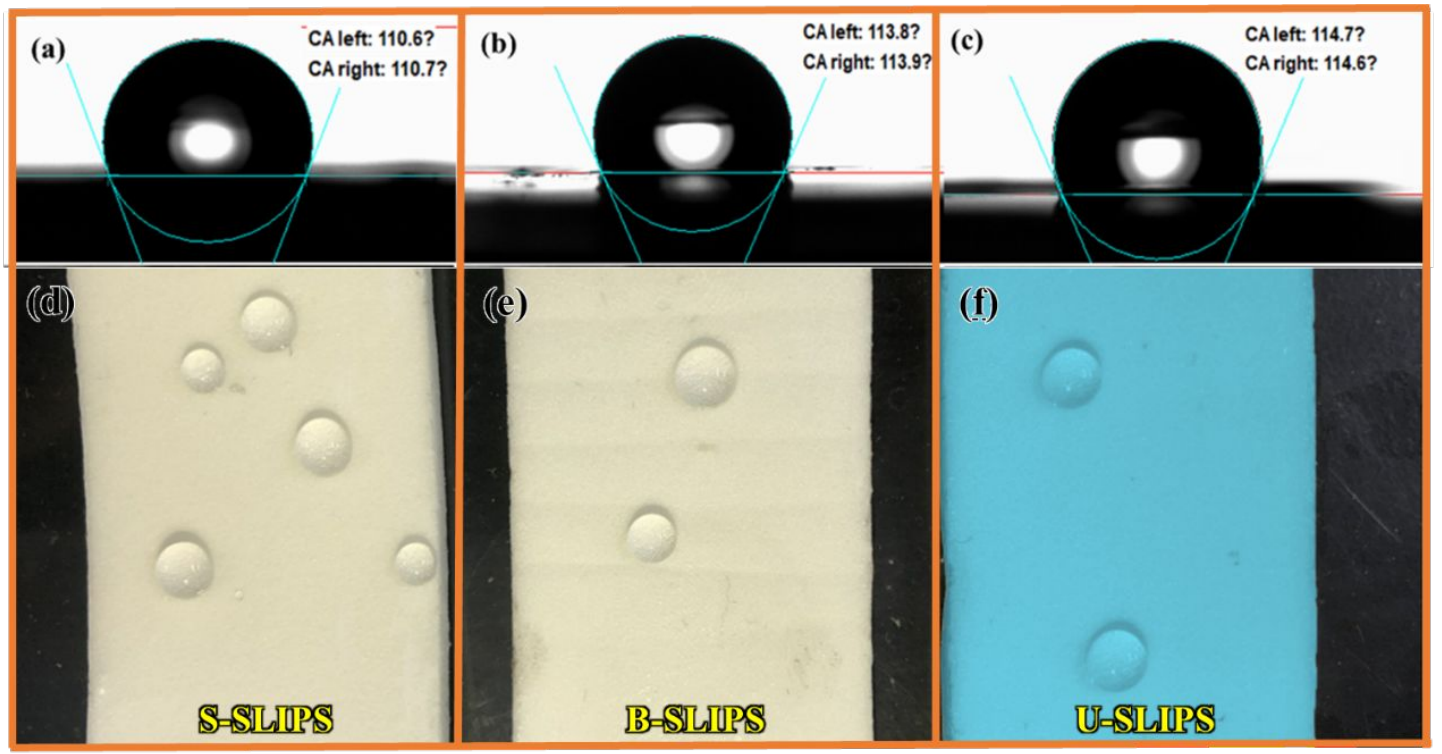

Figure S4 Side view of contact angles of different surfaces (a) S-SLIPS, (b) B-SLIPS,

(c) U-SLIPS. Top view of contact angles of different surfaces (d) S-SLIPS (e) B-SLIPS

(f) U-SLIPS 


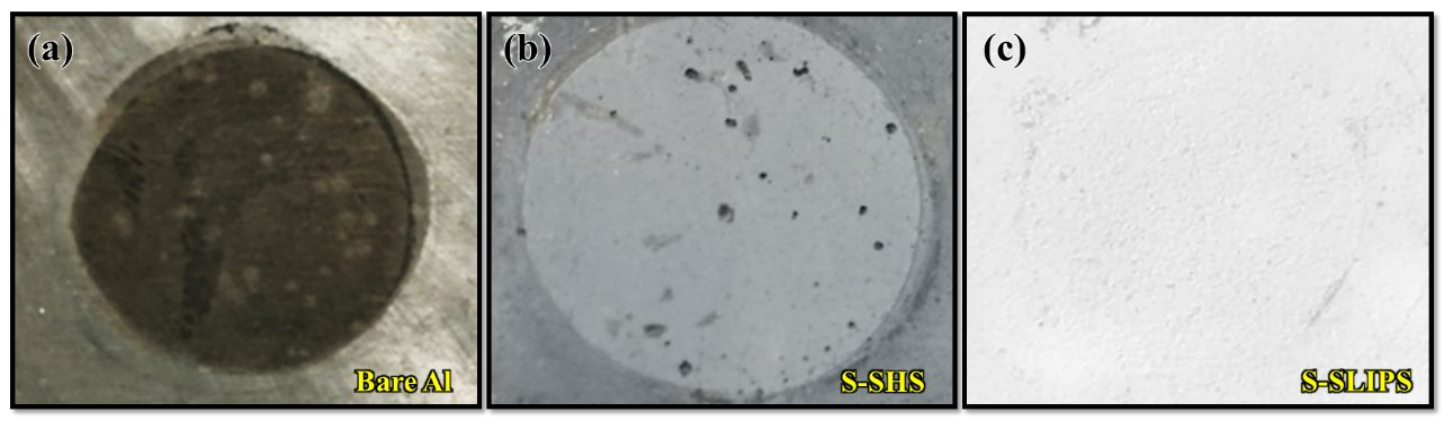

Figure S5 Digital photographs of different surfaces soaked in $3.5 \mathrm{wt} \% \mathrm{NaCl}$ aqueous solution for 47 d. (a) Bare Al alloy, (b)S- SHS, (c) S-SLIPS 


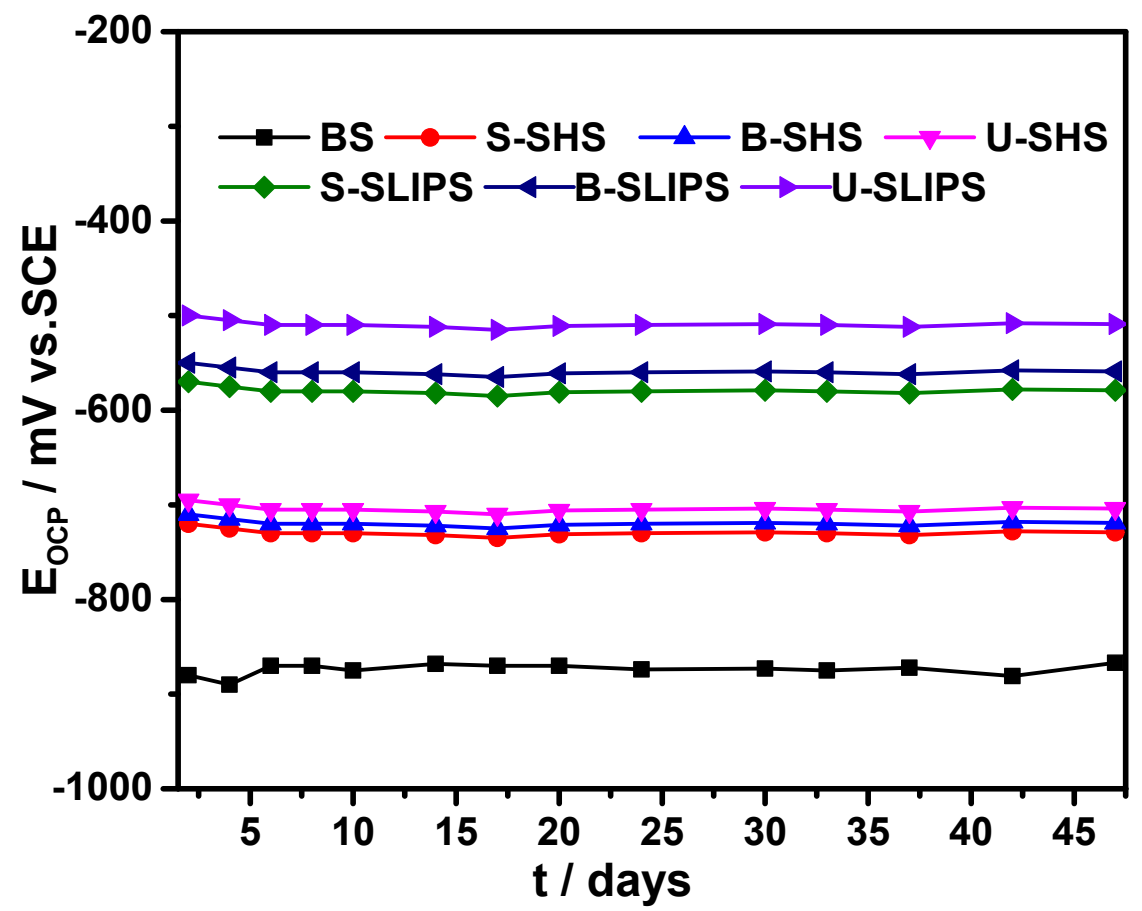

Figure S6 Open-circuit potential (OCP) as a function of exposure time for different samples. The OCP value of BS is around $880 \mathrm{mV}$ vs. SCE, which is more positive than that of SHS and SLIPS. The OCP value of S-SHS, B-SHS and U-SHS are approximately $-725,-718$ and $-698 \mathrm{mV}$ vs. SCE, respectively. The OCP value of SSLIPS, B-SLIPS and U-SLIPS are approximately $-578,-563$ and $-500 \mathrm{mV}$ vs. SCE, respectively. The results indicating that the U-SLIPS presents best stability in $\mathrm{NaCl}$ solution. 

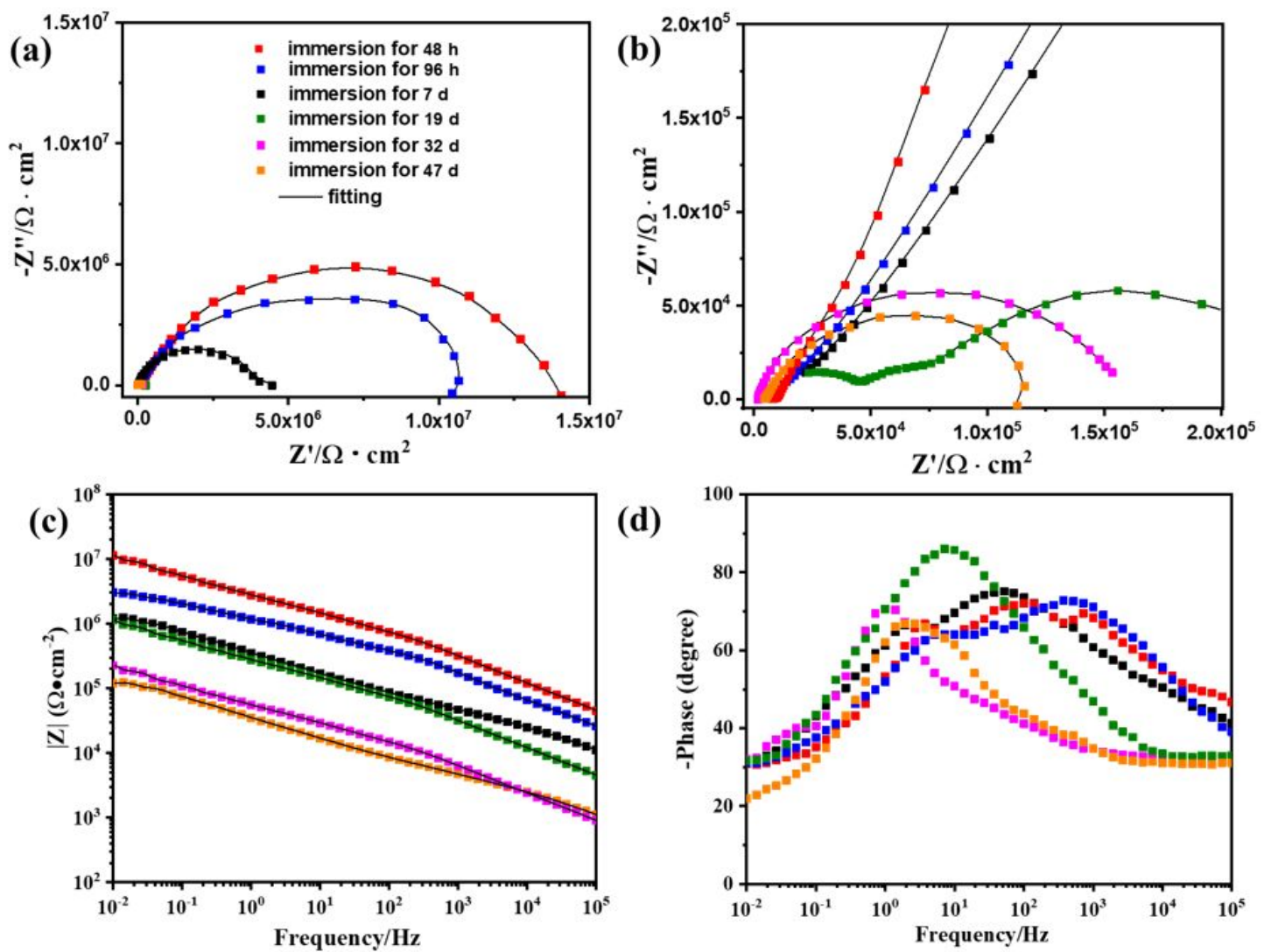

(d)

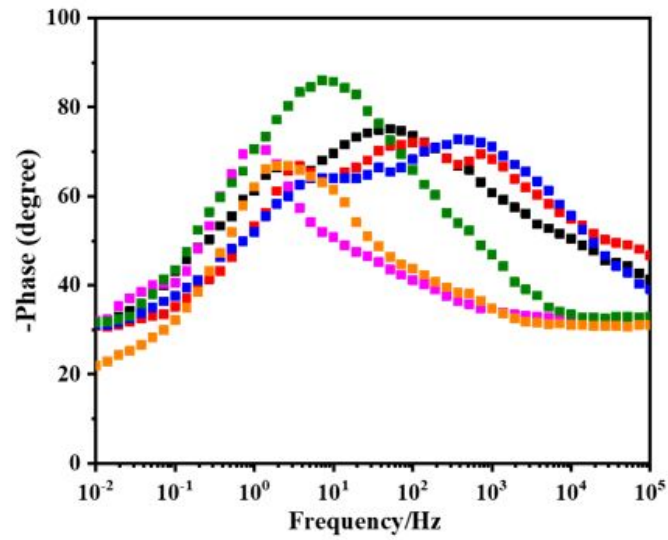

Figure S7 EIS results of barberry-like superhydrophobic surfaces (B-SHS) after immersion in $3.5 \% \mathrm{NaCl}$ solution for different time. (a) Nyquist plots. (b) Partial enlargement of (a). (c) Corresponding Bode plots of $|\mathrm{Z}|$ vs. Frequency. The $|\mathrm{Z}|_{0.01 \mathrm{~Hz}}$ decreases to $10^{5}$ after immersion for 47 d. (d) Corresponding Bode plots of phase angle vs. Frequency. The phase angles maintain a wide range and have high values, which proves that even if the impedance modulus decreases, it still has good protection performance.

Table S1 Electrochemical parameters extracted via fitting EIS of B-SHS.

\begin{tabular}{|c|c|c|c|c|c|c|c|c|c|}
\hline \multirow{2}{*}{$\begin{array}{c}\text { Immersion } \\
\text { time }\end{array}$} & \multicolumn{2}{|c|}{$C P E_{\text {air }}$} & \multirow{2}{*}{$\begin{array}{c}R_{\text {air }} \\
\left(\Omega \cdot \mathbf{c m}^{2}\right)\end{array}$} & \multicolumn{2}{|c|}{$C P E_{\operatorname{mix}}$} & \multirow{2}{*}{$\begin{array}{c}R_{\operatorname{mix}} \\
\left(\Omega \cdot \mathbf{c m}^{2}\right)\end{array}$} & \multicolumn{2}{|c|}{$C P E_{\mathrm{dl}}$} & \multirow{2}{*}{$R_{\mathrm{ct}}\left(\boldsymbol{\Omega} \cdot \mathrm{cm}^{2}\right)$} \\
\hline & $Y_{0}\left(\mathbf{F} / \mathbf{c m}^{2}\right)$ & $n$ & & $Y_{0}\left(\mathrm{~F} / \mathrm{cm}^{2}\right)$ & $n$ & & $Y_{0}\left(\mathrm{~F} / \mathrm{cm}^{2}\right)$ & $n$ & \\
\hline $48 \mathrm{~h}$ & $2.44 \times 10^{-9}$ & 0.82 & $2.01 \times 10^{6}$ & $2.31 \times 10^{-9}$ & 0.81 & $4.11 \times 10^{6}$ & $3.65 \times 10^{-8}$ & 0.72 & $2.07 \times 10^{7}$ \\
\hline $96 \mathrm{~h}$ & $3.26 \times 10^{-9}$ & 0.85 & $3.72 \times 10^{6}$ & $1.98 \times 10^{-8}$ & 0.77 & $3.22 \times 10^{6}$ & $4.30 \times 10^{-7}$ & 0.71 & $1.47 \times 10^{7}$ \\
\hline $7 \mathrm{~d}$ & $1.75 \times 10^{-8}$ & 0.78 & $5.45 \times 10^{6}$ & $4.99 \times 10^{-8}$ & 0.77 & $6.53 \times 10^{5}$ & $2.42 \times 10^{-6}$ & 0.70 & $3.55 \times 10^{6}$ \\
\hline $19 \mathrm{~d}$ & $4.83 \times 10^{-6}$ & 0.79 & $6.00 \times 10^{5}$ & $5.27 \times 10^{-8}$ & 0.79 & $7.63 \times 10^{5}$ & $1.97 \times 10^{-6}$ & 0.69 & $2.53 \times 10^{5}$ \\
\hline $32 \mathrm{~d}$ & $2.77 \times 10^{-6}$ & 0.74 & $5.78 \times 10^{5}$ & $7.55 \times 10^{-7}$ & 0.76 & $1.98 \times 10^{4}$ & $2.63 \times 10^{-5}$ & 0.80 & $1.57 \times 10^{5}$ \\
\hline $47 \mathrm{~d}$ & - & - & - & $3.44 \times 10^{-6}$ & 0.69 & $2.79 \times 10^{4}$ & $4.71 \times 10^{-5}$ & 0.79 & $1.24 \times 10^{5}$ \\
\hline
\end{tabular}



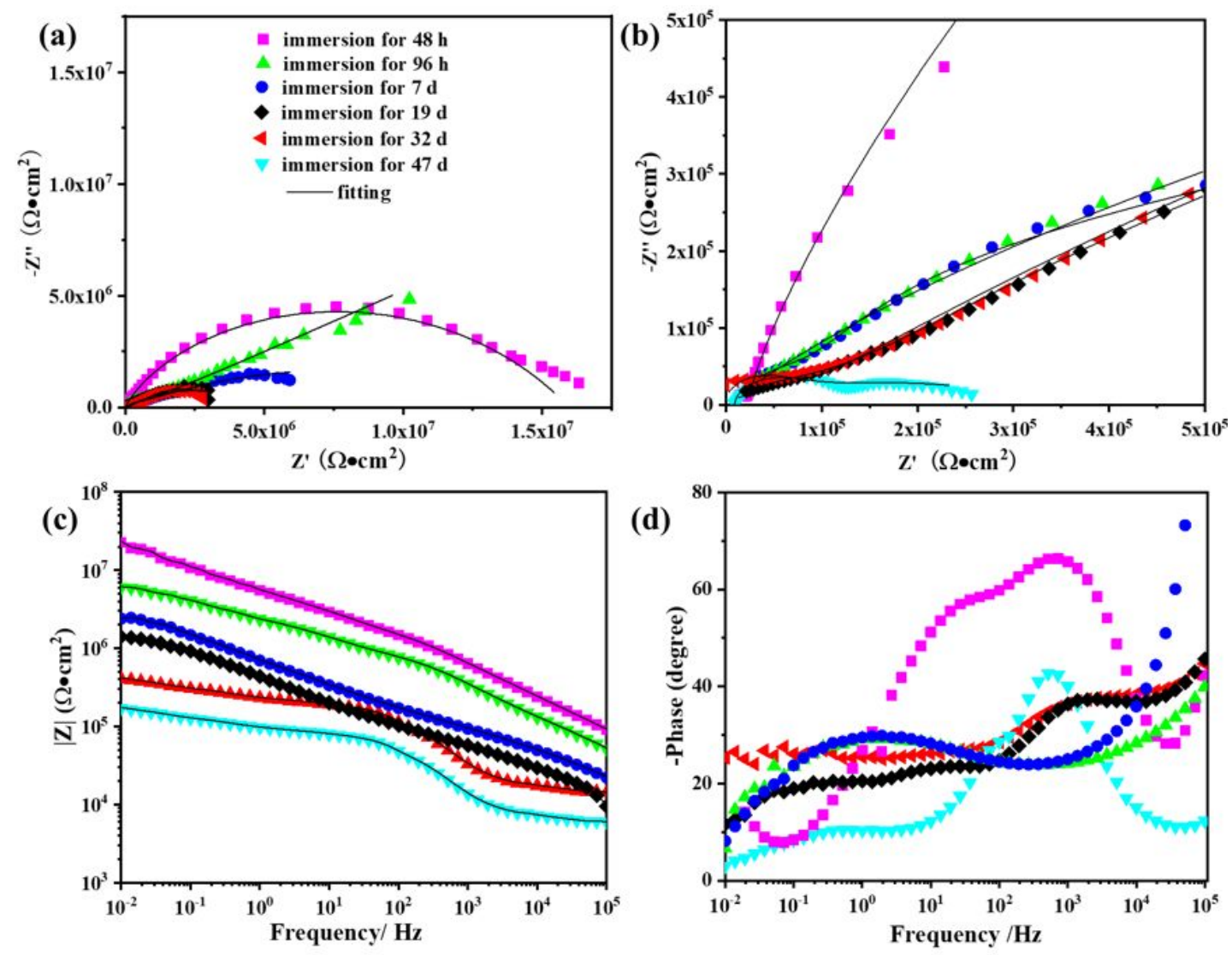

Figure S8 EIS results of $\mathrm{SiO}_{2}$ solid sphere superhydrophobic surfaces (S-SHS) after immersion in $3.5 \% \mathrm{NaCl}$ solution for different time. (a) Nyquist plots. (b) Partial enlargement of (a). (c) Corresponding Bode plots of $|\mathrm{Z}|$ vs. Frequency. The $|\mathrm{Z}|_{0.01 \mathrm{~Hz}}$ decreases to $10^{5}$ after immersion for 47 d, still 2 order of magnitude higher than that of BS. (d) Corresponding Bode plots of phase angle vs. Frequency. After $32 \mathrm{~d}$, there is a time-constant in the low-frequency region which indicating the electrochemical reaction occurs near the interface of coating/substrate

Table S2 Electrochemical parameters extracted via fitting EIS of B-SHS.

\begin{tabular}{|c|c|c|c|c|c|c|c|c|c|}
\hline \multirow{2}{*}{$\begin{array}{c}\text { Immersion } \\
\text { time }\end{array}$} & \multicolumn{2}{|c|}{$C P E_{\text {air }}$} & \multirow{2}{*}{$\begin{array}{c}R_{\text {air }} \\
\left(\Omega \cdot \mathbf{c m}^{2}\right)\end{array}$} & \multicolumn{2}{|c|}{$C P E_{\operatorname{mix}}$} & \multirow{2}{*}{$\begin{array}{c}R_{\text {mix }} \\
\left(\Omega \cdot \mathrm{cm}^{2}\right)\end{array}$} & \multicolumn{2}{|c|}{$C P E_{\mathrm{dl}}$} & \multirow{2}{*}{$R_{\mathrm{ct}}\left(\Omega \cdot \mathrm{cm}^{2}\right)$} \\
\hline & $Y_{0}\left(\mathrm{~F} / \mathrm{cm}^{2}\right)$ & $n$ & & $Y_{0}\left(\mathrm{~F} / \mathrm{cm}^{2}\right)$ & $n$ & & $Y_{0}\left(\mathrm{~F} / \mathrm{cm}^{2}\right)$ & $n$ & \\
\hline $48 \mathrm{~h}$ & $1.94 \times 10^{-8}$ & 0.87 & $2.01 \times 10^{6}$ & $3.47 \times 10^{-7}$ & 0.88 & $4.13 \times 10^{6}$ & $5.31 \times 10^{-8}$ & 0.74 & $1.64 \times 10^{7}$ \\
\hline $96 \mathrm{~h}$ & $5.03 \times 10^{-8}$ & 0.85 & $8.33 \times 10^{5}$ & $3.02 \times 10^{-7}$ & 0.84 & $3.05 \times 10^{6}$ & $1.27 \times 10^{-7}$ & 0.71 & $1.17 \times 10^{7}$ \\
\hline $7 \mathrm{~d}$ & $4.12 \times 10^{-7}$ & 0.88 & $4.23 \times 10^{5}$ & $4.78 \times 10^{-6}$ & 0.83 & $6.77 \times 10^{6}$ & $2.42 \times 10^{-6}$ & 0.68 & $6.24 \times 10^{6}$ \\
\hline $19 \mathrm{~d}$ & $3.16 \times 10^{-6}$ & 0.83 & $6.45 \times 10^{5}$ & $5.39 \times 10^{-6}$ & 0.78 & $5.87 \times 10^{5}$ & $3.00 \times 10^{-6}$ & 0.85 & $1.25 \times 10^{6}$ \\
\hline $32 \mathrm{~d}$ & - & - & - & $5.17 \times 10^{-6}$ & 0.82 & $4.25 \times 10^{5}$ & $4.14 \times 10^{-6}$ & 0.77 & $1.17 \times 10^{6}$ \\
\hline $47 \mathrm{~d}$ & - & - & - & $2.44 \times 10^{-6}$ & 0.80 & $2.44 \times 10^{4}$ & $4.32 \times 10^{-6}$ & 0.84 & $2.75 \times 10^{5}$ \\
\hline
\end{tabular}



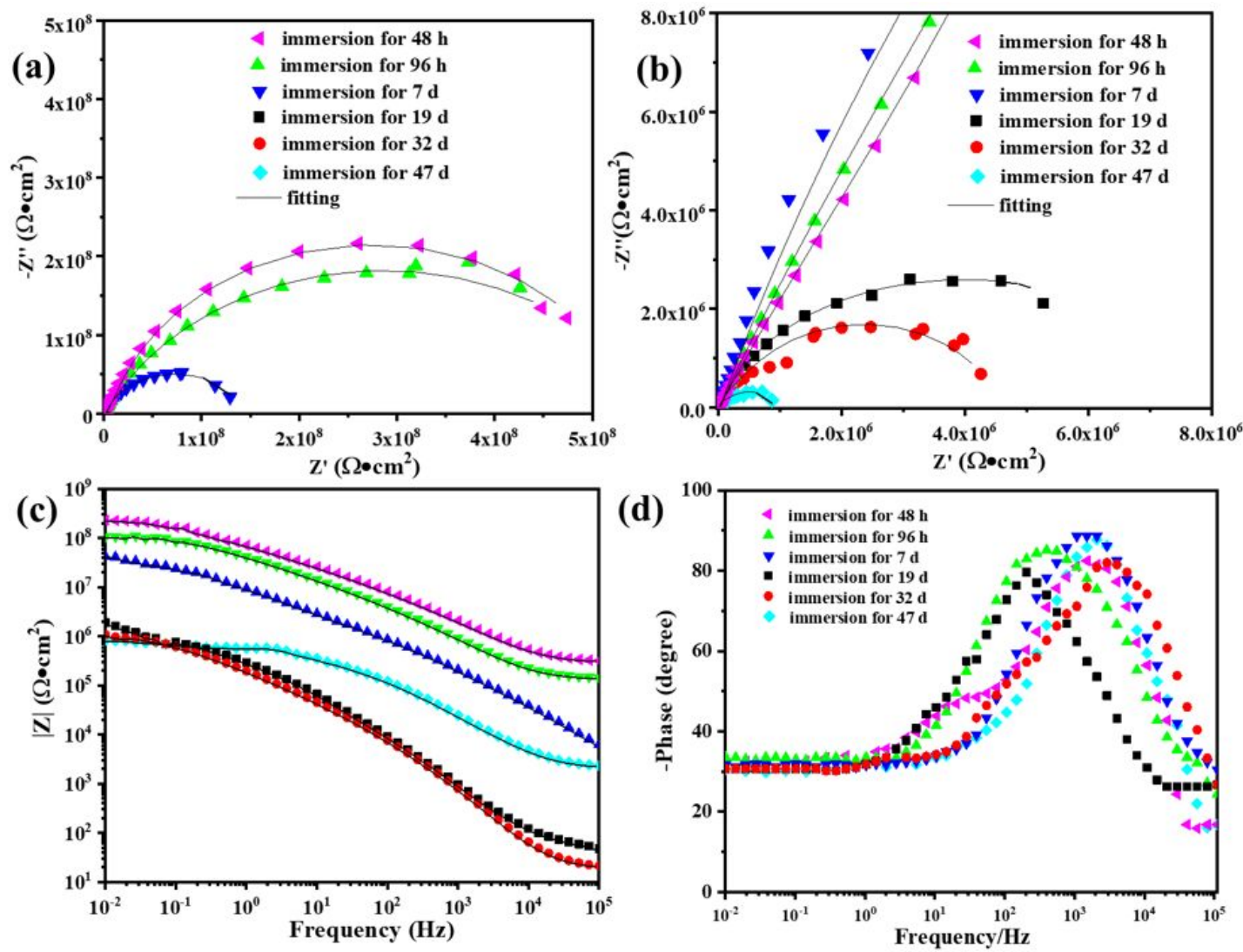

Figure S9. EIS results of barberry-like super slippery surfaces (B-SLIPS) after immersion in 3.5\%

$\mathrm{NaCl}$ solution for different time. (a) Nyquist plots. (b) Partial enlargement of (a). (c) Corresponding Bode plots of $|\mathrm{Z}|$ vs. Frequency. The $|\mathrm{Z}|_{0.01 \mathrm{~Hz}}$ from $5.51 \times 10^{8}$ decreases to $1.11 \times 10^{6}$ even after immersion for 47 d. (d) Corresponding Bode plots of phase angle vs. Frequency. The phase angle has not changed much, which proves that the film has good stability. The results show that the surface has excellent corrosion resistance and long-term protection.

Table S3. The parameter value corresponding to equivalent circuit of B-SLIPS after immersed for different time

\begin{tabular}{|c|c|c|c|c|c|c|c|c|c|}
\hline \multirow{2}{*}{$\begin{array}{c}\text { Immersion } \\
\text { time }\end{array}$} & \multicolumn{2}{|c|}{$C P E_{\text {oil }}$} & \multirow{2}{*}{$\begin{array}{c}R_{\text {oil }} \\
\left(\Omega \cdot \mathrm{cm}^{2}\right)\end{array}$} & \multicolumn{2}{|c|}{$C P E_{\mathrm{mix}}$} & \multirow{2}{*}{$\begin{array}{c}R_{\text {mix }} \\
\left(\Omega \cdot \mathbf{c m}^{2}\right)\end{array}$} & \multicolumn{2}{|c|}{$C P E_{\mathrm{dl}}$} & \multirow{2}{*}{$R_{\text {ct }}\left(\Omega \cdot \mathbf{c m}^{2}\right)$} \\
\hline & $Y_{0}\left(\mathrm{~F} / \mathrm{cm}^{2}\right)$ & $n$ & & $Y_{0}\left(\mathrm{~F} / \mathrm{cm}^{2}\right)$ & $n$ & & $Y_{0}\left(\mathrm{~F} / \mathrm{cm}^{2}\right)$ & $n$ & \\
\hline $48 \mathrm{~h}$ & $1.57 \times 10^{-8}$ & 0.89 & $3.15 \times 10^{7}$ & $3.77 \times 10^{-7}$ & 0.78 & $5.20 \times 10^{6}$ & $3.73 \times 10^{-8}$ & 0.92 & $5.51 \times 10^{8}$ \\
\hline $96 \mathrm{~h}$ & $1.44 \times 10^{-8}$ & 0.82 & $1.87 \times 10^{6}$ & $4.55 \times 10^{-7}$ & 0.83 & $5.24 \times 10^{6}$ & $3.49 \times 10^{-8}$ & 0.93 & $4.83 \times 10^{8}$ \\
\hline $7 \mathrm{~d}$ & $1.56 \times 10^{-7}$ & 0.84 & $1.99 \times 10^{5}$ & $2.27 \times 10^{-7}$ & 0.85 & $5.49 \times 10^{6}$ & $5.47 \times 10^{-8}$ & 0.87 & $1.64 \times 10^{8}$ \\
\hline $19 \mathrm{~d}$ & $1.79 \times 10^{-7}$ & 0.88 & $2.33 \times 10^{5}$ & $1.38 \times 10^{-7}$ & 0.87 & $4.72 \times 10^{6}$ & $5.49 \times 10^{-8}$ & 0.71 & $7.15 \times 10^{6}$ \\
\hline $32 \mathrm{~d}$ & $2.24 \times 10^{-7}$ & 0.88 & $2.18 \times 10^{5}$ & $3.43 \times 10^{-7}$ & 0.86 & $4.38 \times 10^{6}$ & $5.75 \times 10^{-8}$ & 0.78 & $4.21 \times 10^{6}$ \\
\hline $47 \mathrm{~d}$ & $3.41 \times 10^{-7}$ & 0.93 & $8.91 \times 10^{4}$ & $8.46 \times 10^{-7}$ & 0.91 & $4.79 \times 10^{6}$ & $4.16 \times 10^{-7}$ & 0.69 & $1.11 \times 10^{6}$ \\
\hline
\end{tabular}



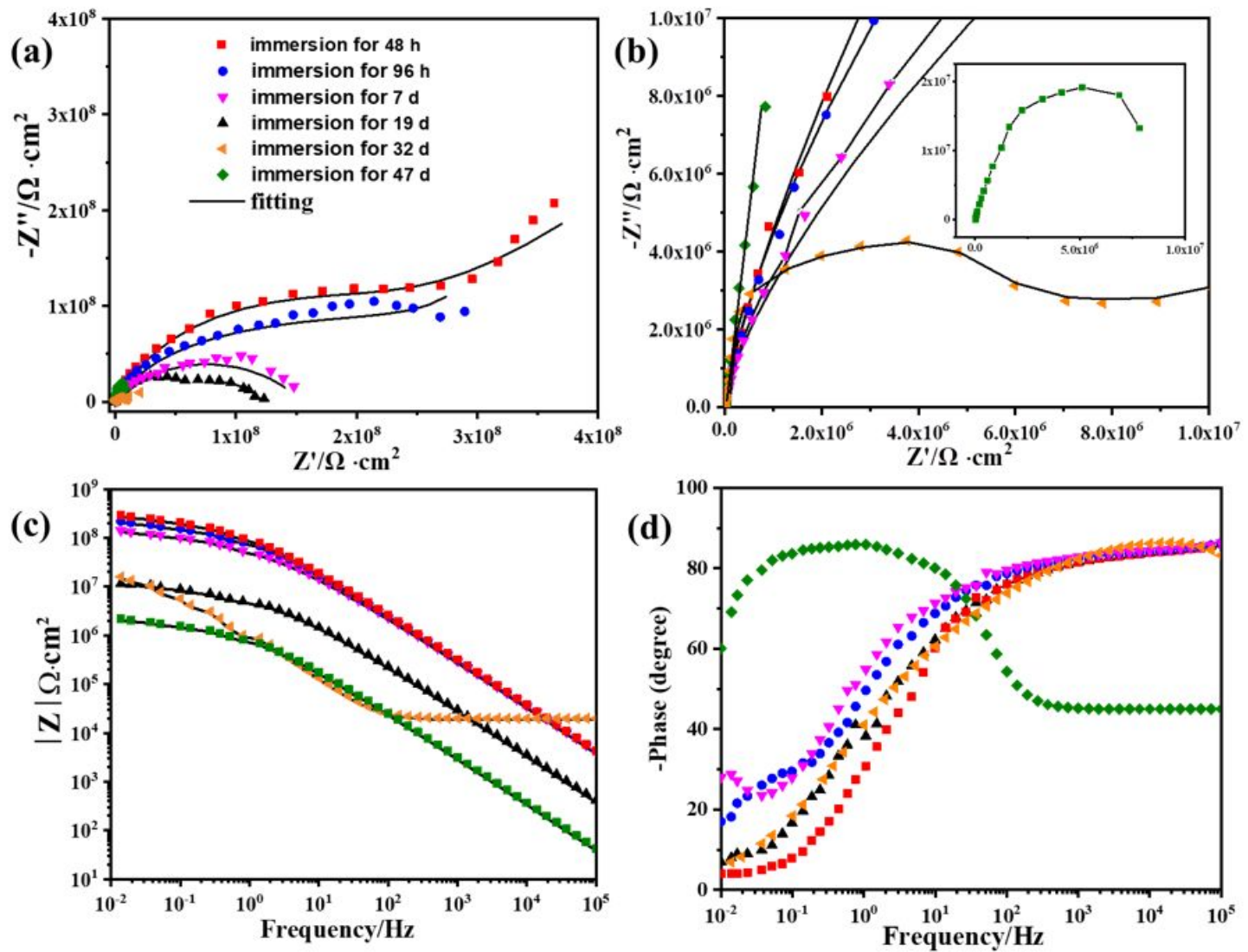

Figure S10 EIS results of $\mathrm{SiO}_{2}$ solid sphere super slippery surfaces (S-SLIPS) after immersion in 3.5\% NaCl solution for different time. (a) Nyquist plots. (b) Partial enlargement of (a). (c) Corresponding Bode plots of $|\mathrm{Z}|$ vs. Frequency. The $|\mathrm{Z}|_{0.01 \mathrm{~Hz}}$ decreases to $10^{6}$ after immersion for 47 d. (d) Corresponding Bode plots of phase angle vs. Frequency. After $32 \mathrm{~d}$, the phase angle position is obviously shifted to the low frequency direction, and the span is narrowed. It indicates that the properties of the film layer have changed.

Table S4. The parameter value corresponding to equivalent circuit of S-SLIPS after immersed for different time

\begin{tabular}{|c|c|c|c|c|c|c|c|c|c|}
\hline \multirow{2}{*}{$\begin{array}{c}\text { Immersion } \\
\text { time }\end{array}$} & \multicolumn{2}{|c|}{$C P E_{\text {oil }}$} & \multirow{2}{*}{$\begin{array}{c}R_{\text {oil }} \\
\left(\Omega \cdot \mathrm{cm}^{2}\right)\end{array}$} & \multicolumn{2}{|c|}{$C P E_{\mathrm{mix}}$} & \multirow{2}{*}{$\begin{array}{c}R_{\text {mix }} \\
\left(\Omega \cdot \mathbf{c m}^{2}\right)\end{array}$} & \multicolumn{2}{|c|}{$C P E_{\mathrm{dl}}$} & \multirow{2}{*}{$R_{\text {ct }}\left(\Omega \cdot \mathbf{c m}^{2}\right)$} \\
\hline & $Y_{0}\left(\mathrm{~F} / \mathrm{cm}^{2}\right)$ & $n$ & & $Y_{0}\left(\mathrm{~F} / \mathrm{cm}^{2}\right)$ & $n$ & & $Y_{0}\left(\mathrm{~F} / \mathrm{cm}^{2}\right)$ & $n$ & \\
\hline $48 \mathrm{~h}$ & $0.95 \times 10^{-8}$ & 0.83 & $4.88 \times 10^{7}$ & $3.77 \times 10^{-7}$ & 0.74 & $4.33 \times 10^{7}$ & $4.44 \times 10^{-8}$ & 0.84 & $3.91 \times 10^{8}$ \\
\hline $96 \mathrm{~h}$ & $1.51 \times 10^{-8}$ & 0.77 & $2.64 \times 10^{7}$ & $4.55 \times 10^{-7}$ & 0.81 & $4.74 \times 10^{7}$ & $4.18 \times 10^{-8}$ & 0.87 & $3.03 \times 10^{8}$ \\
\hline $7 \mathrm{~d}$ & $1.47 \times 10^{-8}$ & 0.81 & $3.17 \times 10^{7}$ & $2.27 \times 10^{-7}$ & 0.82 & $3.52 \times 10^{7}$ & $4.00 \times 10^{-8}$ & 0.83 & $1.52 \times 10^{8}$ \\
\hline $19 \mathrm{~d}$ & $2.02 \times 10^{-8}$ & 0.80 & $2.91 \times 10^{7}$ & $1.38 \times 10^{-7}$ & 0.80 & $2.83 \times 10^{7}$ & $3.75 \times 10^{-8}$ & 0.75 & $1.25 \times 10^{8}$ \\
\hline $32 \mathrm{~d}$ & $1.58 \times 10^{-8}$ & 0.74 & $2.46 \times 10^{6}$ & $3.43 \times 10^{-7}$ & 0.81 & $7.09 \times 10^{6}$ & $4.56 \times 10^{-7}$ & 0.68 & $1.34 \times 10^{7}$ \\
\hline $47 \mathrm{~d}$ & $2.41 \times 10^{-8}$ & 0.79 & $8.91 \times 10^{5}$ & $8.46 \times 10^{-7}$ & 0.79 & $8.69 \times 10^{5}$ & $3.28 \times 10^{-7}$ & 0.71 & $7.77 \times 10^{6}$ \\
\hline
\end{tabular}



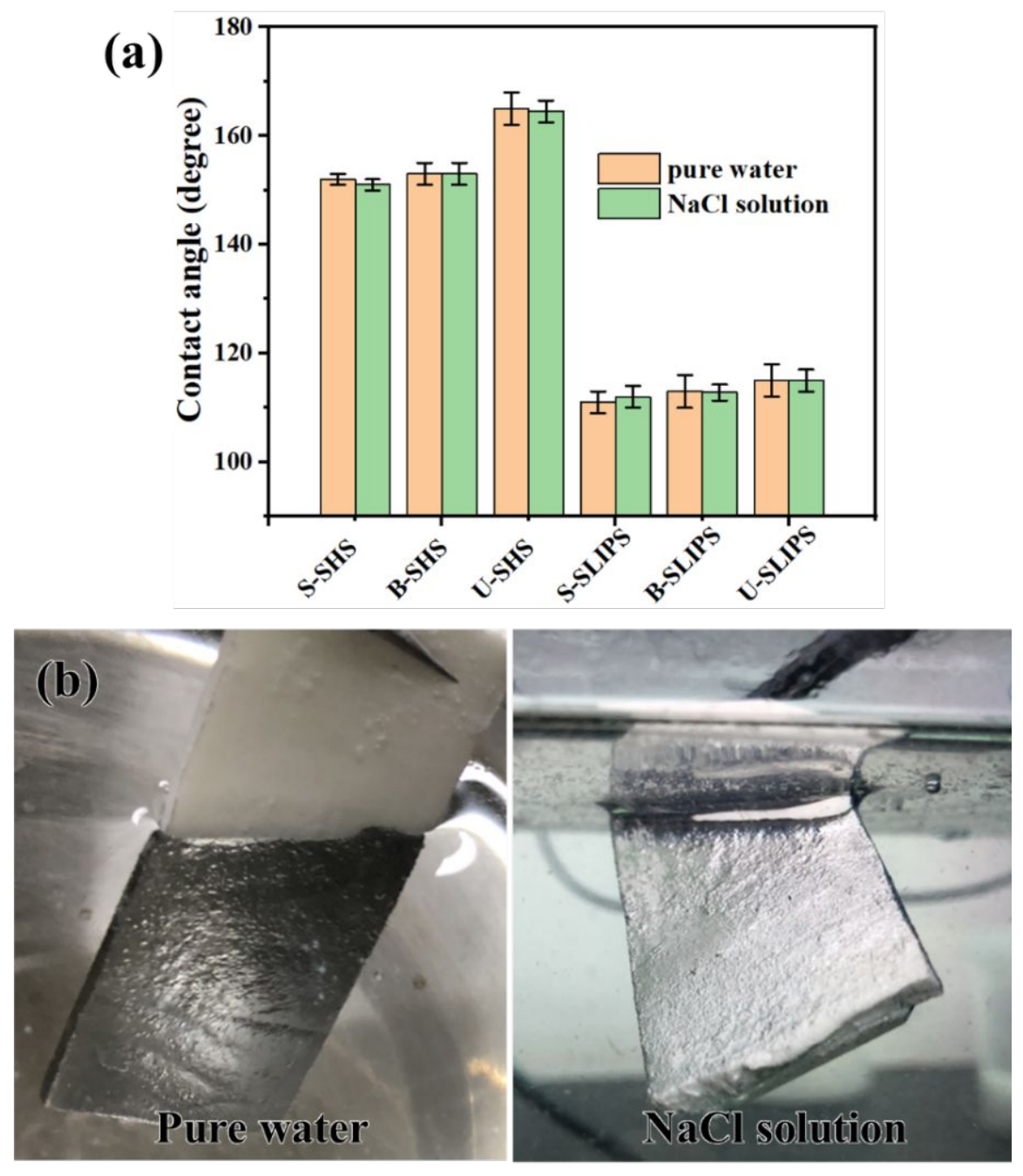

Figure S11 (a) contact angles of different surfaces with different solution. (b) Digital photos of SHS in the pure water and $3.5 \mathrm{wt} . \% \mathrm{NaCl}$ solution. The contact angles of the $\mathrm{NaCl}$ solution droplets are almost the same as that of pure water by testing. As shown in Figure $\mathrm{S} 11 \mathrm{~b}$, the digital photos of B-SHS in the pure water and $3.5 \mathrm{wt} . \% \mathrm{NaCl}$ solution shows the same air layers. 